\title{
Area inequalities for stable marginally outer trapped surfaces in Einstein-Maxwell-dilaton theory
}

\author{
David Fajman and Walter Simon
}

\begin{abstract}
We prove area inequalities for stable marginally outer trapped surfaces in Einstein-Maxwell-dilaton theory. Our inspiration comes on the one hand from a corresponding upper bound for the area in terms of the charges obtained recently by Dain, Jaramillo and Reiris [1] in the pure Einstein-Maxwell case without symmetries, and on the other hand from Yazadjiev's inequality [2] in the axially symmetric Einstein-Maxwell-dilaton case. The common issue in these proofs and in the present one is a functional $\mathscr{W}$ of the matter fields for which the stability condition readily yields an upper bound. On the other hand, the step which crucially depends on whether or not a dilaton field is present is to obtain a lower bound for $\mathscr{W}$ as well. We obtain the latter by first setting up a variational principle for $\mathscr{W}$ with respect to the dilaton field $\phi$, then by proving existence of a minimizer $\psi$ as solution of the corresponding Euler-Lagrange equations and finally by estimating $\mathscr{W}(\psi)$. In the special case that the normal components of the electric and magnetic fields are proportional we obtain the area bound $A \geq 8 \pi P Q$ in terms of the electric and magnetic charges. In the generic case our results are less explicit but imply rigorous 'perturbation' results for the above inequality. All our inequalities are saturated for a 2parameter family of static, extreme solutions found by Gibbons [3]. Via the Bekenstein-Hawking relation $A=4 S$ our results give positive lower bounds for the entropy $S$ which are particularly interesting in the Einstein-Maxwell-dilaton case.
\end{abstract}

\section{Introduction}

Geometric inequalities for MOTS. Marginally outer trapped surfaces (MOTS) are compact 2-surfaces in spacetime on which the orthogonally outgoing family of null geodesics has vanishing expansion. In applications, 'stable' and 'strictly stable' MOTS play an important role and have been 
studied thoroughly [4]-[7]. Here stability is a mild restriction which in essence requires that a certain 3-dim. neighbourhood of the MOTS can be foliated by trapped surfaces inside and untrapped surfaces outside. While the stability condition came up first in connection with the topology theorems for MOTS $[4,8]$, it also plays a key role in problems concerning the time evolution of initial data containing MOTS [9], in particular in some versions of the singularity theorems [10].

Recently there have been proven some remarkable area inequalities for stable MOTS [1], [2], [11]-[14]. They provide bounds for the area $A$ in terms of other naturally available parameters like electric and magnetic charges $P, Q$, the cosmological constant $\Lambda$ and the angular momentum $J$ in the axially symmetric case. The typical general form is $A \geq A_{0}(P, Q, \Lambda, J)$ for some constant $A_{0}$ (but for $\Lambda>0$ there are in addition upper bounds on $A$, cf. Sect. 4.). These inequalities are purely quasilocal in the sense that they involve only the geometry in a neighborhood of the MOTS. In particular they are a priori not related to the more familiar Penrose inequalities (and conjectures) [15] which bound the ADM-mass $M$ from below in terms of the area of a MOTS and other quantities, viz. $M \geq M_{0}(A, P, Q, \Lambda, J)$. On the other hand, the area inequalities obviously provide some sort of 'missing link' between Penrose-type inequalities and 'improved positive mass theorems' ('Bogomolny bounds'; cf.e.g. [16]-[18]) by which we mean inequalities of the form $M \geq M_{0}^{\prime}(P, Q, \Lambda, J)$, which are valid (or conjectured) irrespective of the presence of any MOTS (see also [19]).

MOTS in EMD spacetimes. The derivation of the 'basic' version of the area inequality, which needs suitable matter (-parameters) but no symmetries, is straightforward in principle. If the MOTS has spherical topology (which is guaranteed if the energy-momentum tensor $T_{\alpha \beta}$ satisfies the dominant energy condition) the stability condition implies (cf. Lemma 2.1)

$$
\int_{\mathscr{S}} T_{\alpha \beta} k^{\alpha} \ell^{\beta} d S \leq \frac{1}{2}
$$

Here $k^{\alpha}$ and $\ell^{\beta}$ are future oriented null vectors orthogonal to the MOTS scaled such that $\ell^{\alpha} k_{\alpha}=-1$, and our units are such that Einstein's equations, which have been used to get (1.1), read $G_{\alpha \beta}=8 \pi T_{\alpha \beta}$. Now the remaining task consists of estimating the 'surface energy' (1.1) from below in terms of the available parameters. In this process, the area either comes up automatically or can be brought in naturally. 
In the present work we consider the Einstein-Maxwell-dilaton (EMD) system given by the Lagrangian

$$
\mathscr{L}=\left(R-2 g^{\alpha \beta} \phi_{\alpha} \phi_{\beta}-e^{2 c \phi} F_{\alpha \beta} F^{\alpha \beta}\right)
$$

where $\phi$ is the dilaton, $\phi_{\alpha}=\nabla_{\alpha} \phi$ its gradient, $F_{\alpha \beta}=2 \nabla_{[\alpha} A_{\beta]}$ is the electromagnetic field tensor with vector potential $A_{\beta}$ and $c$ a coupling constant. For this system we can reformulate (1.1) as (cf. Lemma 3.1)

$$
\mathscr{W}(\phi)=\int_{\mathscr{S}}\left(|D \phi|^{2}+e^{-2 c \phi} q^{2}+e^{2 c \phi} p^{2}\right) d S=8 \pi \int_{\mathscr{S}} T_{\alpha \beta} k^{\alpha} \ell^{\beta} \leq 4 \pi
$$

where $D$ is the intrinsic derivative on $\mathscr{S}$. Now $\mathscr{W}(\phi)$ needs to be estimated from below. In the Einstein-Maxwell (EM) case $(\phi=0)$ this gives [1]

$$
A \geq 4 \pi\left(Q^{2}+P^{2}\right)
$$

and some stronger estimate if a cosmological term is considered as well [13].

Main results of the present work. In the presence of the dilaton field our strategy differs substantially from the EM case. We start with minimizing $\mathscr{W}(\phi)$ in (1.3) with respect to $\phi$, and show existence of smooth solutions to the corresponding Euler-Lagrange equations by standard methods, namely via sub- and supersolutions. Depending on how one chooses these latter solutions the subsequent steps are different. We first (in Section 3.1.3) consider the special case where the normal components of the electric and magnetic fields, denoted by $q$ and $p$, are proportional on $\mathscr{S}$, in which case we obtain the bound

$$
A \geq 8 \pi|P Q| .
$$

The latter estimate is in fact saturated for a 2-parameter family of static, extreme black holes with coupling $c=1$ which are members of a 3 -parameter family of static, generic solutions found by Gibbons [3]. As to the case with arbitrary $p$ and $q$, we first derive a rather crude bound (Thm. 3.2) which has the advantage of involving only the charges $Q$ and $P$ as well as $\sup _{\mathscr{S}}|q / p|$ and $\inf _{\mathscr{S}}|q / p|$. We then proceed with a rather sophisticated estimate (Prop. 3.4) which involves some global geometric parameters of $\mathscr{S}$. This inequality implies in particular a quantitative stability statement for (1.5) (Thm. 3.5) in the sense that if $|q / p|$ is almost constant, the resulting inequalities are close to (1.5). 
The axially symmetric case. While our reasoning holds without assuming any symmetries, it certainly applies to the axially symmetric setting. However, in this case the natural and more ambitious goal is to obtain inequalities which explicitly contain the (Komar-) angular momentum $J$ on the r.h.s., and which are still saturated in non-trivial examples. For this task, the basic stability inequality (1.1) is insufficient and has to be replaced by a suitably adapted variational principle. In the EM case [14] obtained

$$
A \geq 4 \pi \sqrt{4 J^{2}+\left(Q^{2}+P^{2}\right)}
$$

with equality in the extreme Kerr-Newman case. (This inequality was proven before in the stationary case [20]). Similarly, for the EMD system (1.2) Yazadjiev [2] showed that

$$
A \geq 8 \pi \sqrt{\left|J^{2}-Q^{2} P^{2}\right|}
$$

which is saturated for the extreme, stationary Kaluza-Klein black holes (coupling $c=\sqrt{3})$ and for the static $(J=0)$ Gibbons solutions mentioned above. This latter example therefore provides some connection with our results.

MOTS thermodynamics. A physical significance of the area inequalities comes from the Bekenstein-Hawking relation $A=4 S$ for the entropy $S$, (provided its validity extends to MOTS, cf e.g. [21, 22]). In any case, the EMD case is of special interest in black hole thermodynamics. In particular, in the extreme limit of that subfamily of the generic Gibbons solutions for which either $Q$ or $P$ vanishes, one approaches a 'black hole of zero area'in fact a singularity - while the surface gravity stays finite. The (sloppy) thermodynamic interpretation of this object is 'a black hole of zero entropy with non-zero temperature', which has sparked some (serious) discussion (see e.g. [23-25]). Our results are relevant for this issue in the sense that they seem to provide strictly positive lower bounds for the entropy. This will be discussed in Section 5 .

Acknowledgement. We are grateful to Piotr Chruściel for helpful comments. W.S. was funded by the Austrian Science Fund (FWF): P 23337-N16.

\section{Preliminaries}

\subsection{Stable MOTS}

Here we consider a smooth, compact, connected spacelike 2 -surface $\mathscr{S}$ in a spacetime $\left(\mathscr{M}, g_{\alpha \beta}\right)$. Let $k^{\alpha}$ and $\ell^{\beta}$ be future directed null vectors defined 
in a neighborhood of $\mathscr{S}$, orthogonal to $\mathscr{S}$ on $\mathscr{S}$, and scaled such that $k^{\alpha} \ell_{\alpha}=-1$. The vector $\ell^{\alpha}$ is called outgoing; its null expansion is defined as

$$
\theta=h^{\alpha \beta} \nabla_{\alpha} \ell_{\beta},
$$

where $h^{\alpha \beta}=\left(g^{\alpha \beta}+2 k^{(\alpha} \ell^{\beta)}\right)$ is the (inverse) metric on $\mathscr{S}$, and a MOTS is a surface $\mathscr{S}$ with $\theta \equiv 0$. To define a stable MOTS with respect to a normal direction $m^{\alpha}[4,5]$ we consider a foliation of a 3-neighborhood of $\mathscr{S}$ in the direction $m^{\alpha}$ by a 1 -parameter family of 2 -surfaces $\mathscr{S}(\lambda)$, and we require that the variation of $\theta$ in direction $m^{\alpha}$ is positive, viz.

$$
\delta_{\gamma m} \theta \geq 0,
$$

where $\gamma>0$ is the lapse-function on $\mathscr{S}$ corresponding to the foliation $\mathscr{S}(\lambda)$.

We now recall the following Lemma $[4,8]$.

Lemma 2.1. For a stable MOTS $\mathscr{S}$,

$$
\begin{aligned}
0 \leq \int_{\mathscr{S}} \gamma^{-1} \delta_{\gamma m} \theta & \leq \int_{\mathscr{S}}\left[\frac{1}{2} R-8 \pi T_{\alpha \beta} \ell^{\alpha} k^{\beta}\right] d S \\
& =4 \pi\left[(1-g)-2 \int_{\mathscr{S}} T_{\alpha \beta} \ell^{\alpha} k^{\beta} d S\right]
\end{aligned}
$$

where $g$ is the genus of $\mathscr{S}$ and $R$ is the scalar curvature of $\mathscr{S}$.

Here the final step of the proof uses the Gauss-Bonnet theorem.

\subsection{Einstein-Maxwell-dilaton theory}

We consider the EMD Lagrangian of the form (1.2) with $\phi, A_{\alpha}$ and $g_{\alpha \beta}$ in the Sobolev space $H^{3}(\mathscr{M})$. Our notation in essence follows Gibbons [3] who denotes the coupling constant by $g$. We can assume $c>0$ without loss of generality because of the symmetry $(c \rightarrow-c, \phi \rightarrow-\phi)$ of the Lagrangian. $c=\sqrt{3}$ is the Kaluza-Klein coupling $[3,26]$.

The field equations obtained from $\mathscr{L}(1.2)$ can be written as

$$
\begin{aligned}
\square \phi & =\frac{c}{2} e^{2 c \phi} F^{\alpha \beta} F_{\alpha \beta} \\
\nabla_{\alpha}\left(e^{2 c \phi} F^{\alpha \beta}\right) & =0 \\
R_{\alpha \beta}-\frac{1}{2} g_{\alpha \beta} R & =8 \pi T_{\alpha \beta}
\end{aligned}
$$


where

$$
8 \pi T_{\alpha \beta}=2\left(\phi_{\alpha} \phi_{\beta}-\frac{1}{2} g_{\alpha \beta} \phi_{\gamma} \phi^{\gamma}\right)+2 e^{2 c \phi}\left(F_{\alpha}^{\gamma} F_{\gamma \beta}-\frac{1}{4} g_{\alpha \beta} F_{\gamma \delta} F^{\gamma \delta}\right)
$$

We next define $q$ and $p$, the normal components on $\mathscr{S}$ of the electric and magnetic fields by

$$
q=e^{2 c \phi} F_{\alpha \beta} \ell^{\alpha} k^{\beta}, \quad p=* F_{\alpha \beta} \ell^{\alpha} k^{\beta}
$$

and for the two-surface $\mathscr{S}$ we define the charges

$$
Q=\frac{1}{4 \pi} \int_{\mathscr{S}} q d S, \quad P=\frac{1}{4 \pi} \int_{\mathscr{S}} p d S
$$

By virtue of (2.5), $Q$ and $P$ are independent of $\mathscr{S}$ which suggests (2.8) as reasonable definitions, even when as below only a fixed MOTS is considered. On the other hand, from the field equations there does not arise any natural definition of a dilaton charge of a 2-surface (see however Eq. (3.7)). The regularity $\left(\phi, A_{\alpha}, g_{\alpha \beta}\right) \in H^{3}(\mathscr{M})$ assumed above guarantees that $q$ and $p$ are in $H^{2}(\mathscr{S})$ which will be used in the proofs of the area inequalities in Section 3.

\subsection{Static solutions}

We recall here first the 3-parameter Reissner-Nordström family in the EM case and then the 3-parameter family of static, spherically symmetric black hole solutions for the coupling $c=1$ found by Gibbons [3] which plays a canonical role in our estimates.

2.3.1. Reissner-Nordström (coupling $c=0$ ). For ease of comparison with the Gibbons solution we write it in non-standard form, namely

$$
\begin{aligned}
\eta & =\frac{Q}{\rho+M}, \quad \chi=\frac{P}{\rho+M}, \\
d s^{2} & =-\lambda d t^{2}+\lambda^{-1}\left[d \rho^{2}+\left(\rho^{2}-L^{2}\right) d \Omega^{2}\right] .
\end{aligned}
$$

Here

$$
\lambda=\frac{\rho^{2}-L^{2}}{(\rho+M)^{2}}
$$


the electric and magnetic potentials $\eta$ and $\chi$ are defined from the vector potential $A_{\alpha}$ via

$$
A_{\alpha}=\left(\eta, A_{i}\right), \quad B_{i}=\epsilon_{i}^{j k} \partial_{j} A_{k} \quad \text { and } \quad \lambda B_{i}=-\partial_{i} \chi
$$

and $M$ and $L^{2}=M^{2}-Q^{2}-P^{2}$ are constants with $L \geq 0$. The coordinate $\rho$ is related to the usual radial coordinate $r$ via $r=\rho+M$. The (outer) horizon $\mathscr{S}$ is located at $\rho=L$; its area and surface gravity are

$$
A=4 \pi(L+M)^{2}, \quad \kappa=\left.\frac{1}{2} \partial_{\rho} \lambda\right|_{\mathscr{S}}=\frac{L}{(L+M)^{2}} .
$$

The extreme solutions are characterized by $\kappa=0=L$; their area (2.14) is

$$
A=4 \pi M^{2}=4 \pi\left(Q^{2}+P^{2}\right)
$$

2.3.2. The Gibbons solution [3] (coupling $c=1$ ). This family is given by

$$
\begin{aligned}
e^{2 \phi} & =\frac{\rho+E}{\rho+F}, \quad \eta=\frac{Q}{\rho+E}, \quad \chi=\frac{P}{\rho+F}, \\
d s^{2} & =-\lambda d t^{2}+\lambda^{-1}\left[d \rho^{2}+\left(\rho^{2}-L^{2}\right) d \Omega^{2}\right] .
\end{aligned}
$$

Here

$$
\lambda=\frac{\rho^{2}-L^{2}}{(\rho+E)(\rho+F)},
$$

the electric and magnetic potentials $\eta$ and $\chi$ are defined as (2.19) except that now the dilaton enters in the definition of $\chi$

$$
A_{\alpha}=\left(\eta, A_{i}\right), \quad B_{i}=\epsilon_{i}{ }^{j k} \partial_{j} A_{k} \quad \text { and } \quad \lambda e^{2 \phi} B_{i}=-\partial_{i} \chi
$$

and $E, F$ and $L^{2}=E^{2}-2 Q^{2}=F^{2}-2 P^{2}$ are constants with $L \geq 0$. The (outer) horizon $\mathscr{S}$ is again located at $\rho=L$; its area and surface gravity are

$$
A=4 \pi(L+E)(L+F), \quad \kappa=\left.\frac{1}{2} \partial_{\rho} \lambda\right|_{\mathscr{S}}=\frac{L}{(L+E)(L+F)} .
$$

The extreme horizon is still characterized by $\kappa=0=L$ with the important proviso that we now have to assume $E=\sqrt{2} Q \neq 0$ and $F=\sqrt{2} P \neq 0$ as the 
area

$$
A=4 \pi|E F|=8 \pi|P Q|
$$

would otherwise vanish. In fact, for $L=0=E B$ there is a lightlike singularity at $\rho=L[25]$, although the surface gravity $\kappa$ in (2.20) can be shown to remain finite in the extreme limit. This fact has stimulated some discussion, in particular regarding the thermodynamic interpretation [23-25], which we recall in Section 5.

\section{Area inequalities}

We first note that (2.7) satisfies the dominant energy condition, whence any stable MOTS has spherical topology (Lemma 2.1). We continue with a Lemma which collects known results and will be key for what follows.

\section{Lemma 3.1.}

1) For the Einstein-Maxwell-dilaton system (1.2) with $\left(\phi, A_{\alpha}, g_{\mu \nu}\right) \in$ $H^{3}(\mathscr{M})$ the condition of stability of a MOTS (cf. Lemma 2.1) implies

$$
\begin{aligned}
\mathscr{W}(\phi, p, q) & =\int_{\mathscr{S}}\left(|D \phi|^{2}+e^{-2 c \phi} q^{2}+e^{2 c \phi} p^{2}\right) d S \\
& =8 \pi \int_{\mathscr{S}} T_{\alpha \beta} k^{\alpha} \ell^{\beta} \leq 4 \pi
\end{aligned}
$$

where $D^{\alpha}=h^{\alpha \beta} \nabla_{\beta}$ is the intrinsic derivative on $\mathscr{S}$.

2) Considered as a functional of $\phi$, a unique minimizer $\psi \in H^{4}(\mathscr{S})$ of $\mathscr{W}(\phi)$ always exists, satisfies the Euler-Lagrange equations

$$
\Delta \psi=c p^{2} e^{2 c \psi}-c q^{2} e^{-2 c \psi}
$$

and yields the estimate

$$
4 \pi \geq \mathscr{W}(\phi) \geq \mathscr{W}(\psi)=\int_{\mathscr{S}}\left[(1+c \psi) e^{-2 c \psi} q^{2}+(1-c \psi) e^{2 c \psi} p^{2}\right] d S
$$

Moreover, if $\psi_{ \pm} \in H^{4}(\mathscr{S})$ are sub-and supersolutions of (3.2), i.e.

$$
\Delta \psi_{-} \geq c p^{2} e^{2 c \psi_{-}}-c q^{2} e^{-2 c \psi_{-}}, \quad \Delta \psi_{+} \leq c p^{2} e^{2 c \psi_{+}}-c q^{2} e^{-2 c \psi_{+}}
$$

then $\psi_{-} \leq \psi \leq \psi_{+}$. 


\section{Proof.}

1) This part follows by inserting (2.7) in Lemma 2.1 and treating the terms with the electromagnetic fields via Lemma 3.4 of [1].

2) Existence of solutions of (3.2) and the bounds (3.4) for $\psi$ follow from standard results (Thm. 1.10 and Prop. 1.9 of [27]), while (3.3) is obtained by partial integration.

Before proceeding with the general discussion of this equation we now discuss three special cases. While the first two just use the first part of Lemma 3.1 and direct estimates of (1.3), the final one is non-trivial in the sense that it makes use of the second part of Lemma 3.1 as well, in particular it uses an (albeit trivial) solution of (3.2).

\subsection{Special cases}

3.1.1. Einstein-Maxwell. In the pure Einstein-Maxwell case $(\phi=0)$, the Cauchy-Schwarz estimates $\left\langle q^{2}\right\rangle \geq 4 \pi Q^{2}$ and $\left\langle p^{2}\right\rangle \geq 4 \pi P^{2}$ turn (3.1) into [1]

$$
16 \pi^{2} \frac{Q^{2}+P^{2}}{A} \leq \mathscr{W}(\phi) \leq 4 \pi
$$

which gives (1.4); the bound is saturated for the extreme Reissner-Nordström solutions.

3.1.2. Massless scalar field. In the case $F_{\alpha \beta}=0$ we obtain formally the same result

$$
16 \pi^{2} \frac{Z^{2}}{A} \leq \mathscr{W}(\phi) \leq 4 \pi
$$

in terms of a 'dilaton charge'

$$
Z=\|D \phi\|_{L^{1}}=\frac{1}{4 \pi} \int_{\mathscr{S}}|D \phi| d S .
$$

Note however that, in contrast to $Q$ and $P$ which are defined as integrals over the normal components of the electric and magnetic fields, $Z$ is defined from the tangential components $D \phi$. Therefore $Z$ vanishes for all spherically symmetric solutions, no examples are known in which (3.6) is saturated, 
while asymptotically flat static black holes do not exist anyway [28]. Moreover, the integral is surface dependent, whence $Z$ will in general not coincide with dilaton charges defined in the asymptotic region of asymptotically flat solutions. We anticipate that the quantity (3.7) will not appear in any of the estimates derived below.

3.1.3. The case $\boldsymbol{p}=\boldsymbol{\alpha} \boldsymbol{q}$. Here we assume that $p$ and $q$ are proportional with some constant $\alpha \neq 0$. As mentioned above, we now employ (3.2) which becomes

$$
\Delta \psi=c q^{2}\left(\alpha^{2} e^{2 c \psi}-e^{-2 c \psi}\right) .
$$

This is obviously solved by $e^{2 c \psi}=|\alpha|^{-1}$, and it is in fact the unique minimizer by virtue of Proposition 1.9 of [27] which is implicit in Lemma 3.1. Inserting now in (3.3) and using as above the Cauchy-Schwarz estimate yields

$$
A \geq 8 \pi|P Q| .
$$

This bound is saturated for the extreme Gibbons solutions (but not for extreme Reissner-Nordström), and consistent with Yazadjiev's inequality (1.7).

We note that uniqueness of $e^{2 c \psi}=|\alpha|^{-1}$, can also be seen directly rather than by using Lemma 3.1. Namely, integrating (3.8) over $\mathscr{S}$ implies that the expression in parenthesis, if nonzero, changes sign on $\mathscr{S}$. In particular it is positive at the maximum of $\psi$ and negative at a minimum of $\psi$. However, this contradicts the maximum principle and completes the argument.

\subsection{A sup-inf-estimate}

Here we assume that the normal components $q$ and $p$ of the electric and magnetic fields do not vanish on $\mathscr{S}$, and we remark that this entails $Q \neq 0$ and $P \neq 0$. We define the quantities

$$
e^{2 c \mu_{-}}=\inf _{\mathscr{S}}\left|\frac{q}{p}\right|, \quad e^{2 c \mu_{+}}=\sup _{\mathscr{S}}\left|\frac{q}{p}\right|
$$

which play a key role in the following theorem. Note that $\mu_{-} \leq \mu_{+}$since we assumed $c \geq 0$. 
Area inequalities for stable marginally outer trapped surfaces ‥ 697

Theorem 3.2. For the Einstein-Maxwell-dilaton system (1.2) with ( $\phi, A_{\mu}$, $\left.g_{\mu \nu}\right) \in H^{3}(\mathscr{M})$ we consider a smooth, stable MOTS $\mathscr{S}$ and assume that $p \neq 0$ and $q \neq 0$ on $\mathscr{S}$.

Its area satisfies

$$
A \geq 4 \pi\left(N_{q} Q^{2}+N_{p} P^{2}\right)
$$

where

$$
\begin{aligned}
& N_{q}=\min \left[\left(1+c \mu_{-}\right) e^{-2 c \mu_{-}},\left(1+c \mu_{+}\right) e^{-2 c \mu_{+}}\right] \\
& N_{p}=\min \left[\left(1-c \mu_{-}\right) e^{2 c \mu_{-}},\left(1-c \mu_{+}\right) e^{2 c \mu_{+}}\right] .
\end{aligned}
$$

The bound (3.11) is saturated for the extreme Gibbons solutions (2.20).

The proof will be based on Lemma 2.1, in particular on Eq. (3.3), for which we need the following lemma.

Lemma 3.3. The integrand $I(\psi)$ of (3.3), considered as function of $\psi$, has precisely one critical point in the interval $(-\infty, \infty)$ which is a maximum.

Proof. A straightforward calculation shows

$$
\begin{aligned}
I^{\prime}(\psi) & =(1-2 c \psi) p^{2} e^{2 c \psi}-(1+2 c \psi) q^{2} e^{-2 c \psi} \\
I^{\prime \prime}(\psi) & =-4 c^{2} \psi\left(p^{2} e^{2 c \psi}-q^{2} e^{-2 c \psi}\right)
\end{aligned}
$$

and it is easy to see that $I^{\prime \prime}<0$ at critical points which are located at

$$
\frac{p^{2}}{q^{2}} e^{4 c \psi}=\frac{1+2 c \psi}{1-2 c \psi}
$$

A closer inspection shows that there is in fact precisely one such maximum.

Proof of Theorem 3.2. We first note that the constants $\mu_{-}$and $\mu_{+}$defined in (3.10) can serve as sub- and supersolutions in Lemma 3.1. In fact we have

$$
\begin{aligned}
& \Delta \mu_{-}=0 \geq c p^{2} e^{2 c \mu_{-}}-c q^{2} e^{-2 c \mu_{-}}, \\
& \Delta \mu_{+}=0 \leq c p^{2} e^{2 c \mu_{+}}-c q^{2} e^{-2 c \mu_{+}} .
\end{aligned}
$$

Hence by Lemma 3.1 there is a minimizer $\psi \in H^{4}(\mathscr{S})$ which satisfies $\mu_{-} \leq$ $\psi \leq \mu_{+}$. 
To estimate (3.3) from below, we use Lemma 3.3 which implies that, for any point of $\mathscr{S}$, the integrand $I(\psi)$ in (3.3) takes its minimum for one of the boundary values $\mu_{-}$or $\mu_{+}$. We now replace $I(\psi)$ by the minimal boundary value. A subtlety here is that it will in general depend on the point on $\mathscr{S}$ at which of the values $\mu_{-}$or $\mu_{+}$the minimum is taken. We therefore define a partition of $\mathscr{S}$ in terms of two subsets $\mathscr{S}_{-}$and $\mathscr{S}_{+}$with

$$
\mathscr{S}_{-} \cap \mathscr{S}_{+}=\emptyset, \quad \mathscr{S}_{-} \cup \mathscr{S}_{+}=\mathscr{S}
$$

such that on $\mathscr{S}_{ \pm}, I$ takes its minimum at $\mu_{ \pm}$. Note that the definition of $\mathscr{S}_{ \pm}$is not unique if there is a subset $\mathscr{S}_{0} \subset \mathscr{S}$ for which the minima $I\left(\mu_{-}\right)$ and $I\left(\mu_{+}\right)$coincide - in this case $\mathscr{S}_{0}$ can be distributed arbitrarily among $\mathscr{S}_{ \pm}$. Inserting in (1.3) then yields

$$
\begin{aligned}
4 \pi \geq \mathscr{W}(\psi)= & \int_{\mathscr{S}}\left[(1+c \psi) e^{-2 c \psi} q^{2}+(1-c \psi) e^{2 c \psi} p^{2}\right] d S \\
\geq & \int_{\mathscr{S}_{-}}\left[\left(1+c \mu_{-}\right) e^{-2 c \mu_{-}} q^{2}+\left(1-c \mu_{-}\right) e^{2 c \mu_{-}} p^{2}\right] d S \\
& +\int_{\mathscr{S}_{+}}\left[\left(1+c \mu_{+}\right) e^{-2 c \mu_{+}} q^{2}+\left(1-c \mu_{+}\right) e^{2 c \mu_{+}} p^{2}\right] d S \\
\geq & 4 \pi \min \left[\left(1+c \mu_{-}\right) e^{-2 c \mu_{-}},\left(1+c \mu_{+}\right) e^{-2 c \mu_{+}}\right]\left\langle q^{2}\right\rangle \\
& +4 \pi \min \left[\left(1-c \mu_{-}\right) e^{2 c \mu_{-}},\left(1-c \mu_{+}\right) e^{2 c \mu_{+}}\right]\left\langle p^{2}\right\rangle \\
\geq & \frac{16 \pi^{2}}{A}\left(N_{q} Q^{2}+N_{p} P^{2}\right)
\end{aligned}
$$

where here and henceforth we use the notation

$$
\langle f\rangle=\frac{1}{4 \pi} \int_{\mathscr{S}} f d S
$$

for the average of a quantity $f \in L^{2}(\mathscr{S})$, and we have as before used CauchySchwarz estimates in the final step.

An easy exercise is to verify that when $\mu_{-}$approaches $\mu_{+},(3.11)$ tends to (3.9). A more concrete statement will be given in Theorem 3.5 in connection with the subsequent estimate.

\subsection{An $L^{2}$-estimate}

Proposition 3.4. For the Einstein-Maxwell-dilaton system (1.2) with ( $\phi$, $\left.A_{\mu}, g_{\mu \nu}\right) \in H^{3}(\mathscr{M})$ we consider a smooth, stable MOTS $\mathscr{S}$. 
We denote by $\lambda_{1}=\lambda_{1}(\mathscr{S})$ the first non-zero eigenvalue of the LaplaceBeltrami operator on $\mathscr{S}$, and we use the notation $\hat{f}=f / \sqrt{\left\langle f^{2}\right\rangle}$ for a quantity $f \in L^{2}(\mathscr{S})$.

Then there exists a function $\varphi \in H^{2}(\mathscr{S})$ with

$$
\|\varphi\|_{\infty} \leq C\left(\lambda_{1}, \inf _{x \in \mathscr{S}} R\right) \sqrt{\left\langle p^{2}\right\rangle\left\langle q^{2}\right\rangle} \sqrt{\left\langle\left(\hat{p}^{2}-\hat{q}^{2}\right)^{2}\right\rangle}
$$

such that

$$
\begin{aligned}
\mathscr{W} \geq & \sqrt{\left\langle p^{2}\right\rangle\left\langle q^{2}\right\rangle}\left[\int_{\mathscr{S}} e^{-2 c \varphi} \hat{q}^{2}+e^{2 c \varphi} \hat{p}^{2} d S\right. \\
& +\frac{1}{4} \ln \left(\left\langle q^{2}\right\rangle /\left\langle p^{2}\right\rangle\right) \int_{\mathscr{S}}\left(e^{-2 c \varphi} \hat{q}^{2}-e^{2 c \varphi} \hat{p}^{2}\right) d S \\
& \left.+c \int_{\mathscr{S}} \varphi\left(e^{-2 c \varphi} \hat{q}^{2}-e^{2 c \varphi} \hat{p}^{2}\right) d S\right]
\end{aligned}
$$

holds, where $C=C\left(\lambda_{1}, \inf _{x \in \mathscr{S}} R\right)$ is a constant depending on the geometry of $\mathscr{S}$. Moreover, if $\hat{q}=\hat{p}$ this reduces to $\mathscr{W} \geq 32 \pi^{2}|P Q| / A$ which is saturated for the Gibbons solutions (2.20).

A straightforward consequence of Proposition 3.4 is the following "perturbation' of inequality (3.9).

Theorem 3.5. Under the assumptions of Proposition 3.4, for all $\varepsilon>0$ there exists a $\delta>0$ such that for $\left\langle\left(\hat{p}^{2}-\hat{q}^{2}\right)^{2}\right\rangle<\delta$,

$$
A \geq 8 \pi(1-\varepsilon)|P Q| \text {. }
$$

Note that in contrast to Theorem 3.2, $q$ and $p$ are allowed to have zeros on $\mathscr{S}$ in Proposition 3.4 and Theorem 3.5. For $\delta=0$, we have $p=\alpha q$ and the result reduces to $(3.1 .3)$.

Proof of Proposition 3.4. We set out from Lemma 2.1 but instead of taking constant sub- and supersolutions as in Theorem 3.2, we follow the method used by Choquet-Bruhat and Moncrief in another context [29]. Consider the equation

$$
0=e^{2 c \omega}\left\langle p^{2}\right\rangle-e^{-2 c \omega}\left\langle q^{2}\right\rangle
$$

for a real number $\omega$, yielding

$$
\left\langle q^{2}\right\rangle\left\langle p^{2}\right\rangle^{-1}=e^{4 c \omega} .
$$


Then the linear equation

$$
\Delta v=e^{2 c \omega} p^{2}-e^{-2 c \omega} q^{2}=f(\omega)
$$

has a unique solution $v \in H^{4}(\mathscr{S})$ since $\int_{\mathscr{S}} f(\omega)=0$. Sub- and supersolutions are now defined in terms of $v$ by

$$
\psi_{+}=v-\min v+\omega \quad \text { and } \quad \psi_{-}=v-\max v+\omega
$$

which implies (3.4). By Lemma 2.1, there exists a unique solution $\psi \in$ $H^{4}(\mathscr{S})$ to $(3.2)$, with

$$
\mathscr{W}(\zeta) \geq \mathscr{W}(\psi), \quad \forall \zeta \in H^{1}(\mathscr{S})
$$

and

$$
\psi_{-} \leq \psi \leq \psi_{+}
$$

For $\varphi$ defined by

$$
\varphi=\omega-\psi
$$

(3.32) and (3.34) imply the straightforward pointwise estimate

$$
\|\varphi\|_{L^{\infty}(\mathscr{S})} \leq 2\|v\|_{L^{\infty}(\mathscr{S})}
$$

To estimate the minimizer $\psi$ we will now estimate $v$ in terms of geometric quantities. As $v$ is a solution of (3.31), partial integration yields

$$
\int|\nabla v|^{2} d S=-\int v f(\omega) .
$$

which in turn gives

$$
\|\nabla v\|_{L^{2}}^{2} \leq\|f(\omega)\|_{L^{2}}\|v\|_{L^{2}}
$$

The Poincaré inequality for $v$-as a function with mean value zero-reads

$$
\|v\|_{L^{2}}^{2} \leq \frac{1}{\lambda_{1}(g)}\|\nabla v\|_{L^{2}}^{2}
$$

With (3.38) this now implies 


$$
\|\nabla v\|_{L^{2}} \leq \frac{1}{\sqrt{\lambda_{1}(g)}}\|f(\omega)\|_{L^{2}}
$$

Finally, the Ricci identity

$$
\left\|\nabla^{2} z\right\|_{L^{2}}^{2}=\|\Delta z\|_{L^{2}}^{2}-\frac{1}{2} \int R|\nabla z|^{2}, \quad \forall z \in H^{2}(S)
$$

gives

$$
\left\|\nabla^{2} v\right\|_{L^{2}}^{2} \leq\|f(\omega)\|_{L^{2}}^{2}+\frac{\inf R}{2} \int|\nabla v|^{2} d S .
$$

Combining (3.39), (3.40) and (3.42) yields the estimate for $v$

$$
\|v\|_{H^{2}} \leq \sqrt{1+\frac{1}{\lambda_{1}(g)^{2}}\left(1+\lambda_{1}(g)\right)+\frac{\inf R}{2 \lambda_{1}(g)}}\|f(\omega)\|_{L^{2}} .
$$

We note that if scalar curvature $R$ is non-negative on $\mathscr{S}$, the term with $\inf R$ can be dropped from (3.42) and hence from (3.43). Now Sobolev embedding with optimal constant $C(g)$ yields

$$
\|v\|_{\infty} \leq C(g) \sqrt{1+\frac{1}{\lambda_{1}(g)^{2}}\left(1+\lambda_{1}(g)\right)+\frac{\inf R}{2 \lambda_{1}(g)}}\|f(\omega)\|_{L^{2}}
$$

which is (3.26). Next, $f(\omega)$ can be estimated as follows

$$
\begin{aligned}
\|f(\omega)\|_{L^{2}}^{2} & =4 c^{2} \int_{\mathscr{S}}\left(e^{2 c \omega} p^{2}-e^{-2 c \omega} q^{2}\right)^{2} d S \\
& =4 c^{2}\left\langle q^{2}\right\rangle\left\langle p^{2}\right\rangle\left\langle\left(\hat{p}^{2}-\hat{q}^{2}\right)^{2}\right\rangle .
\end{aligned}
$$


Eventually, using (3.3) and resubstituting $\psi=\varphi+\omega$ yields

$$
\begin{aligned}
\mathscr{W} \geq \int & -2 c(\omega+\varphi)\left(p^{2} e^{2 c(\omega+\varphi)}-q^{2} e^{-2 c(\omega+\varphi)}\right) \\
& +p^{2} e^{2 c(\omega+\varphi)}+q^{2} e^{-2 c(\omega+\varphi)} d S \\
= & \sqrt{\left\langle p^{2}\right\rangle\left\langle q^{2}\right\rangle}\left[\int e^{-2 c \varphi} \hat{q}^{2}+e^{2 c \varphi} \hat{p}^{2} d S\right. \\
& +\frac{1}{2} \ln \left(\langle q\rangle^{2} /\langle p\rangle^{2}\right) \int\left(\hat{q}^{2} e^{-2 c \varphi}-\hat{p}^{2} e^{2 c \varphi}\right) d S \\
& \left.+2 c \int \varphi\left(\hat{q}^{2} e^{-2 c \varphi}-\hat{p}^{2} e^{2 c \varphi}\right) d S\right]
\end{aligned}
$$

which was claimed in $(3.27)$

We recall that, compared to Yazadjiev's result (1.7), our theorem does not require axial symmetry. In the axially symmetric case, however, our estimate (3.28) gives complementary infomation whose relevance clearly depends on the relative magnitude of the quantities $|P Q|, J^{2}$ and $\left\langle\left(\hat{p}^{2}-\right.\right.$ $\left.\left.\hat{q}^{2}\right)^{2}\right\rangle$.

\section{The cosmological case}

A class of Lagrangians with cosmological constant with arbitrary dilaton coupling constant $d$, namely

$$
\mathscr{L}=\left(R-2 g^{\alpha \beta} \phi_{\alpha} \phi_{\beta}-e^{2 c \phi} F_{\alpha \beta} F^{\alpha \beta}-\Lambda e^{-d \phi}\right)
$$

has been discussed recently (cf e.g. [30]). It seems interesting to generalize the results for stable MOTS obtained above to this case, but this lies beyond the scope of the present paper. We rather restrict ourselves here to the case $d=0$ for which the generalization is straightforward. As Einstein's equations now read

$$
G_{\alpha \beta}+\Lambda g_{\alpha \beta}=8 \pi T_{\alpha \beta}
$$


Area inequalities for stable marginally outer trapped surfaces $\cdots \quad 703$

the stability condition (1.3) has to be replaced by

$$
\begin{aligned}
\mathscr{W}(\phi) & =\int_{\mathscr{S}}\left(|D \phi|^{2}+e^{-2 c \phi} q^{2}+e^{2 c \phi} p^{2}\right) d S \\
& =\int_{\mathscr{S}}\left(8 \pi T_{\alpha \beta}-\Lambda g_{\alpha \beta}\right) k^{\alpha} \ell^{\beta} \\
& \leq 4 \pi+\Lambda A .
\end{aligned}
$$

This leads to the following quadratic inequality for $A$ as generalization of Theorem 3.2

$$
\Lambda A^{2}-4 \pi(1-g) A+16 \pi^{2}\left(N_{q} Q^{2}+N_{p} P^{2}\right) \leq 0
$$

where $g$ is the genus of $\mathscr{S}$. This inequality can be discussed along the lines of [13]; for $\Lambda>0$ there arise an upper and a lower bound for $A$, as well as the upper bound $N_{q} Q^{2}+N_{p} P^{2} \leq 1 /(4 \Lambda)$. On the other hand, for $\Lambda<0$, there is just a lower bound for the area.

In a similar manner Theorem 3.5 now reads that for all $\varepsilon>0$ there exists a $\delta>0$ such that for $\left\langle\left(\hat{p}^{2}-\hat{q}^{2}\right)^{2}\right\rangle<\delta$,

$$
\Lambda A^{2}-4 \pi(1-g) A+32 \pi^{2}(1-\varepsilon)|P Q| \leq 0
$$

with area bounds as before; for $\Lambda>0$ the upper bound on the charges now reads $(1-\varepsilon)|P Q| \leq 1 /(2 \Lambda)$.

\section{Black holes versus elementary particles}

As indicated in the introduction and in Section 2.3.2, static EMD black holes have interesting thermodynamic properties in the extreme limit. We first recall the cases in which solutions are known explicitly.

\subsection{Arbitrary $c$ but either electric or magnetic field vanishing}

In this case there is the 2-parameter family of solutions found in [31] which contains an extreme 1-parameter subfamily. The area of the horizons goes to zero in the extreme limit and for all $c \neq 0$. More precisely, this family approaches a singular solution where the singularity was found to be lightlike [25]. As to the surface gravity $\kappa$, the value $c=1$ is critical in the sense that for $c<1, \kappa$ goes to zero in the extreme limit, for $c=1$ it approaches a constant, while for $c>1$ it diverges. 


\subsection{Coupling $c=0$ or $c=1$, and arbitrary Maxwell field}

Here there are the 3-parameter families of Reissner-Nordström and Gibbons solutions with 2-parameter extreme subfamilies, which have been reviewed in Section 2.3.2. For the Gibbons solutions $(c=1)$ we have seen that the area satisfies $A=8 \pi|P Q|$; in particular, it stays positive in the extreme limit provided that neither charge vanishes.

Of course the thermodynamic interpretation of the extreme limit is subtle when the zero area $\widehat{=}$ zero entropy $\widehat{=}$ singular solution with non-zero surface gravity $\widehat{=}$ temperature is approached. Based on perturbation analyses with axially symmetric, time dependent perturbations and with a spherically symmetric, time dependent test field, Holzhey and Wilczek [23] have argued that for $c=1$ the static extremal solutions (5.1) enjoy a finite 'mass gap' in the sense that these objects are repulsive for low-energy perturbations, while for $c>1$ the mass gap is infinite and the object is universally repulsive. Accordingly, these authors put forward an analogy between these configurations and elementary particles.

In the static, spherically symmetric examples discussed above, the 'particle-like' behaviour is associated with singular solutions having 'zero horizon area' and therefore zero entropy. If this connection persists in nonsymmetric, dynamical spacetimes (in the dynamical case, the proportionality between entropy and area has been under dispute [21, 22]), Yazadjiev's [2] and our theorems provide trivial criteria to judge potential 'particle candidates': The former results, which apply to stable axially symmetric MOTS, single out configurations with $|P Q|=|J|$ as good candidates since $A=0$, while they exclude those with $|P Q| \neq|J|$ for which $A>0$. On the other hand, in the generic case our Theorems 3.2 and 3.5 exclude several configurations with stable MOTS, again by guaranteeing that $A>0$. In particular, Theorem 3.5 excludes solutions for which P.Q $\neq 0$ and for which $q$ and $p$ are either proportional or close to being proportional.

\section{References}

[1] S. Dain, J. L. Jaramillo and M. Reiris, Area-charge inequality for black holes, Class. Quantum Grav. 29035013 (2012).

[2] S. Yazadjiev, Area-angular momentum-charge inequality for stable marginally outer trapped surfaces in 4 D Einstein-Maxwell-dilaton theory, Phys. Rev. D 87024016 (2013). 
Area inequalities for stable marginally outer trapped surfaces $\cdots 705$

[3] G. Gibbons, Antigravitating black hole solitons with scalar hair in $N=$ 4 supergravity, Nucl. Phys. B 207337 (1982).

[4] R. Newman, Topology and stability of marginal 2-surfaces, Class. Quantum Grav. 4277 (1987).

[5] L. Andersson, M. Mars and W. Simon, Stability of marginally outer trapped surfaces and existence of marginally outer trapped tubes, Adv. Theor. Math. Phys. 12853 (2008).

[6] L. Andersson, M. Eichmair and J. Metzger, Jang's Equation and Its Applications to Marginally Trapped Surfaces, Proceedings of the Fourth International Conference on Complex Analysis and Dynamical Systems (M. Agranovsky [et al.] eds.), Contemporary Mathematics 553, American Mathematical Society and Bar-Ilan University, (2009).

[7] J. L. Jaramillo, An introduction to local black hole horizons in the $3+1$ approach to general relativity, Int. J. of Modern Physics 202169 (2011).

[8] G. Galloway, Constraints on the topology of higher dimensional black holes, Black Holes in Higher Dimensions (G. T. Horowitz ed.), Cambridge University Press, (2012).

[9] L. Andersson, M. Mars, J. Metzger and W. Simon, The time evolution of marginally trapped surfaces, Class. Quantum Grav. 26085018 (2008).

[10] I. P. Costa e Silva, On the geodesic incompleteness of spacetimes containing marginally (outer) trapped surfaces, Class. Quantum Grav. 29 235008 (2012).

[11] S. Dain, Geometric inequalities for axially symmetric black holes, Class. Quantum Grav. 29073001 (2012).

[12] J. L. Jaramillo, Area Inequalities for Stable Marginally Trapped Surfaces, Springer Proceedings in Mathematics \& Statistics 26139 (2013).

[13] W. Simon, Bounds on area and charge for marginally trapped surfaces with a cosmological constant, Class. Quantum Grav. 29062001 (2012).

[14] M. E. Gabach Clement, J. L. Jaramillo and M. Reiris, Proof of the areaangular momentumcharge inequality for axisymmetric black holes, Class. Quantum Grav. 30065017 (2013).

[15] M. Mars, Class. Present status of the Penrose inequality, Quantum Grav. 26, 193001 (2009). 
[16] R. Bartnik and P. Chruściel, Boundary value problems for Dirac-type equations, J. Reine Angew. Math. 579, 13 (2005).

[17] P. Chrusciel and J. L. Costa, Mass, angular-momentum, and charge inequalities for axisymmetric initial data, Class. Quant. Grav. 26 235013 (2009).

[18] R. Schoen and X. Zhou, Convexity of Reduced Energy and Mass Angular Momentum Inequalities, Ann. Henri Poincaré 14, 1747 (2013).

[19] S. Dain, M. Khuri, G. Weinstein and S. Yamada, Lower bounds for the area of black holes in terms of mass, charge, and angular momentum, Phys. Rev. D 88, 024048 (2013).

[20] J. Hennig, C. Cederbaum and M. Ansorg, A Universal Inequality for Axisymmetric and Stationary Black Holes with Surrounding Matter in the Einstein-Maxwell Theory, Commun. Math. Phys. 293, 449 (2010).

[21] A. Corichi and D. Sudarsky, When is $S=A / 4$ ? Mod. Phys. Lett. A 17, 1431 (2002).

[22] A. Nielsen, Black holes without Boundaries, Int. J. Mod. Phys. D 17, 2359 (2009).

[23] C. Holzhey and F. Wilczek, Black holes as elementary particles, Nucl. Phys. B 380447 (1992).

[24] J. H. Horne and G. T. Horowitz, Rotating dilaton black holes, Phys Rev. D 46, 1340 (1992).

[25] G. Horowitz, What is the true description of charged black holes? Directions in General Relativity, Vol. 2 (B. L. Hu and T. A. Jacobson eds.), Cambridge University Press, (1993).

[26] G. Horowitz and T. Wiseman, General black holes in KaluzaKlein theory, Black Holes in Higher Dimensions (G. T. Horowitz ed.), Cambridge University Press, (2012).

[27] M. Taylor, Partial Differential Equations III, Springer, New York, (1996).

[28] J. Chase, Event horizons in static scalar-vacuum space-times, Commun. Math. Phys. 19, 276 (1970).

[29] Y. Choquet-Bruhat and V. Moncrief, Future Global in Time Einsteinian Spacetimes with U(1) Isometry Group, Ann. Henri Poincaré 21007 (2001). 
Area inequalities for stable marginally outer trapped surfaces $\cdots \quad 707$

[30] C. Charmousis, D Langlois, D Steer and R. Zegers, Rotating spacetimes with a cosmological constant, JHEP 02(2007):064.

[31] G. Gibbons and K. Maeda, Black holes and membranes in higherdimensional theories with dilaton fields, Nucl. Phys. B 298741 (1988).

Gravitational Physics, Faculty of Physics, University of Vienna Boltzmanngasse 5, 1090 Vienna, Austria

E-mail address: David.Fajman@univie.ac.at

Gravitational Physics, Faculty of Physics, University of Vienna

Boltzmanngasse 5, 1090 Vienna, Austria

E-mail address: Walter.Simon@univie.ac.at 
